\title{
ROAD TRAFFIC NOISE AND ANNOYANCE: EXPOSURE- RESPONSE RELATIONSHIP AND BURDEN OF DISEASE CALCULATIONS IN BULGARIA
}

\author{
Angel Dzhambov ${ }^{1}$, Donka Dimitrova ${ }^{2}$ \\ ${ }^{1}$ Faculty of Medicine, Medical University of Plovdiv, \\ ${ }^{2}$ Department of Health Management, Health Economics and Primary Care, \\ Faculty of Public Health, Medical University of Plovdiv
}

\begin{abstract}
BACKGROUND: Noise annoyance (NA) contributes considerably to the cumulative burden of disease from environmental noise in Europe. Exposure-response functions are the basis for assessment of its socioeconomic impact which is essential for environmental health policy. These functions have not been implemented in Bulgaria.

AIM: In order to determine the socio-economic impact of road traffic NA in Plovdiv and Bulgaria several objectives were set: to establish a preliminary exposure-response relationship between road traffic noise and NA; to compare it to Miedema and Oudshoorn's curve recommended by the World Health Organization (WHO); and to quantify the burden of high NA.

MATERIAL AND METHODS: Based on data collected during a survey on cardio-metabolic environmental risk factors in Plovdiv we generated an exposure-response function derived from a logistic model and compared it to Miedema and Oudshoorn's polynomial. Employing the official WHO methodology we calculated the disability-adjusted life years (DALYs) lost in Plovdiv due to high NA and monetized the value of these DALYs. Then we extended the analyses to the total Bulgarian urban population.
\end{abstract}

RESULTS: The logistic function in our study bared close resemblance to Miedema and Oudshoorn's polynomial (range 55-75 dB). Based on the latter, an annual loss of 1 188.17 DALYs was estimated in Plovdiv which translated into $€ 14.26$ million assuming $€ 12000$ per DALY. On a national scale the results were even more alarming (6 399.60 DALYs and $€ 76.80$ million).

CONCLUSION: Road traffic NA appears to have a considerable socio-economic impact in the country which calls for decisive actions by involved authorities. Future use of Miedema and Oudshoorn's polynomial is substantiated.

Keywords: road traffic noise, noise annoyance, exposure-response relationship, burden of disease, disability-adjusted life-years, environmental economics

Address for correspondence:

Angel Dzhambov

Faculty of Medicine, Medical University of Plovdiv

15-A Vasil Aprilov Blvd.

4002 Plovdiv, Bulgaria

e-mail:angelleloti@gmail.com

Received: April 06, 2015

Accepted: June 8, 2015

\section{INTRODUCTION}

Traffic noise contributes substantially to the cumulative burden of disease in Europe (1). Its liaisons with various cardio-metabolic and psycho-social health outcomes (2) make it a prominent environmental pollutant, second only to air pollution (1). One of the most studied reactions to noise is annoyance defined as "a multifaceted psychological con- 
Angel Dzhambov, Donka Dimitrova

cept, covering immediate behavioural noise effects aspects, like disturbance and interfering with intended activities, and evaluative aspects like 'nuisance', 'disturbance', 'unpleasantness', and 'getting on one's nerves' (3). Noise annoyance (NA) is not only a proxy for the actual noise level, but it encompasses psychological reactions to it as well. About a third of the variance in NA is explained by the noise exposure levels, another third by psycho-social and cultural factors and the last third remains unexplained (4).

A meta-analysis found 16\% (95\% CI: 1.02, 1.29) higher odds for arterial hypertension and 7\% (0.99, 1.14) for ischemic heart disease among highly annoyed individuals (5). Conservatively, 587000 disability-adjusted life years (DALYs) are lost annually to NA in Western Europe (1). Bulgaria, however, lacks national-level data regarding the socio-economic costs of NA. Given the high preponderance of people exposed to unacceptably high noise levels (6) and the regular complaints about traffic noise by citizens of major Bulgarian agglomerations (7), one can expect that these costs will be overwhelming.

Population-based socio-acoustic surveys are not always practical. Environmental noise policy is contingent on generalized exposure-response curves derived from meta-analyses to estimate the proportion of "highly annoyed" (\% HA) people across different noise bands as a function of noise, which is an important anchor element of environmental impact analyses allowing to take substantiated political action (1). At individual level various non-acoustical factors are largely influential determinants of NA (8), but the relative importance of individual differences is reduced on a macro-scale (9), whereas the predictive power of noise exposure raises up to $70 \%$ (10), making this ecological approach feasible.

Despite the growing concerns in Bulgaria about road traffic noise as a community nuisance we lack validated exposure-response curves describing this relationship. Miedema and Oudshoorn hypothesized that the reaction to noise across populations in different countries should not differ substantially (9) but the functions they reported were derived from studies carried out in high-income countries; both the social and environmental context and the quality of noise mapping might be sufficiently different in Bulgaria to affect the exposure-response functions despite the uniform requirements for noise mapping and the reduced importance of individual factors at a group level. Thus the need is felt for addressing these uncertainties and filling the gap in environmental noise research in the country.

This study aimed to determine the socio-economic impact of road traffic NA in Plovdiv and the country. Several objectives were set: to establish a preliminary exposure-response relationship between road traffic noise and NA in the population; to compare it to the standard curve used by the World Health Organization (WHO); and to estimate the burden of severe NA based on the latter.

\section{MATERIAL AND METHODS}

This research comprised two stages: first, a small-scale field survey on the environmental risk factors for cardio-metabolic health in Plovdiv was carried out which allowed us to construct an exposure-response function between road traffic noise and NA and to compare it to the officially recommended function by the WHO; as a next step, both functions were applied to the official noise exposure data for Plovdiv and Bulgaria in order to quantify the burden of severe NA and its economic valuation.

\section{Stage 1: Field survey}

Design

A small-scale epidemiological survey was carried out in Plovdiv (July - November, 2014), one of the most noise and air polluted cities in Bulgaria (6, 11). The respondents were selected via two methods a non-probability snowball sampling and a field sampling. The present study uses data from the later in order to ensure some generalizability of our results.

Current residency in Plovdiv and being over 18 years of age were inclusion criteria whereas people reporting uncorrected hearing impairment were excluded from the study. Field sampling started with the selection of 41 neighbourhood blocks on an interactive city map based on their variability in road traffic, noise, air pollution and environmental greenness. The first author visited those neighbourhoods and approached people in local green spaces, in the street, in front of their homes, in local shopping centres, etc. The interviews took about 10 minutes. Due to lack of external funding, no financial incentives were offered. 
Because of its non-interventional nature and adult population the study did not undergo ethics approval by the University Committee. All participants were assured of anonymity. Answering the questionnaire was taken to constitute informed consent.

\section{Questionnaire and variables}

A 59-item questionnaire was administered. The following self-reported variables were relevant to the present study:

* Demographics: age, sex, ethnicity ("Bulgarian", "other"), highest educational level attained ("basic", "upper secondary", "bachelor/master", "PhD/DSc"), marital status ("married/with a spouse", "single", "widowed", “divorced”), occupation ("employed", "studying", "unemployed", "retired") and socio-economic status ("lower", "middle", "upper").

* Noise sensitivity was measured with the Noise Sensitivity Questionnaire Short Form (NoiSeQSF) (12).

* The degree of NA was elicited by means of an 11 -point scale (" 0 , not at all" to " 10 , very much") similar to the one recommended by ISO 15666 (cited by ref. 13). Scoring $>7$ (i.e., 8,9 or 10) was used as a cut-off indicating high NA. The measure, however, did not specify the source of NA. Road traffic noise assessment

Participants answered a question about their address in Plovdiv. Exact addresses were geocoded using BatchGeocode (https://en.batchgeo.com/) and Google Earth ${ }^{\mathrm{TM}}$. When participants had provided only the name of the street they lived at, the addresses were matched to the centre point of the street (14). A direct inspection of each reported address/residential street was performed (Google Street View). When possible, the building of residence was identified based on the reported description. Relevant exposure data were assigned after overlaying road traffic noise maps onto the map of geocoded addresses.

In compliance with the Environmental Noise Directive the member states prepare strategic noise maps following harmonized guidelines (15). The recommended noise indicator for overall annoyance that we used is the $\mathrm{L}_{\text {den }}$ (day-evening-night equivalent sound level) - A-weighted annual noise level measured over 24 hours, with a $10 \mathrm{~dB}$ penalty added to night-time and a $5 \mathrm{~dB}$ penalty to evening hours. The noise map was downloaded from the website of $\mathrm{Mu}$ nicipality of Plovdiv (http://www.plovdiv.webnoise. eu/strategicheska_karta_en.php).

Field survey data analysis and computation of exposure-response function

Initial descriptive statistics were computed. Data were screened for univariate normality and outliers using D'Agostino-Pearson $\mathrm{K}^{2}$ test and a modification of the outlier labelling rule. Cases with missing data were subjected to listwise deletion in order to minimize possible bias given the objectives of the study. Pearson's ( $r$ ) and Spearman's $\left(r_{s}\right.$ ) correlation coefficients were applied to NA depending on the type of the other variables. The $95 \%$ confidence intervals $(95 \% \mathrm{CI})$ for the two types of correlation coefficients were calculated applying the same computational procedure for both (17). Welch's t-tests and ANOVAs tested the difference in NA scores across categorical factors. NA was treated as a continuous variable.

The \%HA people in each $\mathrm{L}_{\text {den }}$ noise band was plotted along with its 95\% CI against the respective mid-category noise level. The 95\% CI was calculated according to Wilson's procedure without a continuity correction (18).

A logistic model was set up in order to generate a function describing the single exposure-response relationship between NA and $\mathrm{L}_{\text {den }}$. $\mathrm{L}_{\text {den }}$ was treated as a continuous independent variable assuming that the mid-category value represented reasonably well each $5-\mathrm{dB}$ noise band. The probability of high NA was estimated with the following logistic fit:

$$
\% H A=\frac{e^{(\beta 0+\beta 1 * X i)}}{1+e^{(\beta 0+\beta 1 * X i)}}
$$

where $\beta_{0}$ and $\beta_{1}$ are the intercept and the slope of the logistic response function.

Ninety five \% CI and 95\% prediction interval (95\% PI) of the logistic prediction curve were graphed according to the following formulae:

$$
95 \% C I=Y \pm t_{\alpha} * S E \sqrt{\frac{1}{n}+\frac{\left(X-X_{m}\right)^{2}}{S S_{X X}}}
$$

and 


$$
95 \% P I=Y \pm t_{\alpha} * S E \sqrt{1+\frac{1}{n}+\frac{\left(X-X_{m}\right)^{2}}{S S_{X X}}}
$$

where $\mathrm{Y}-\% \mathrm{HA}, \mathrm{t}_{\alpha}$ - Student's $\mathrm{t}$ for $\alpha=0.05, \mathrm{SE}$ - standard error, $\mathrm{n}$ - number of cases, $\mathrm{X}$ - average of all $\mathrm{L}_{\text {den }}$ values, $\mathrm{X}_{\mathrm{m}}$ - specific $\mathrm{L}_{\text {den }}$ value, $\mathrm{SS}_{\mathrm{Xx}}$ - sum of squares (explained variance).

The logistic prediction curve along with its linear approximation were compared to the polynomial reported by Miedema and Oudshoorn (9) which is recommended by the WHO for assessment of NA due to road traffic noise:

$$
\% H A=9.868^{\star} 10^{-4 *}\left(L_{d e n}-42\right)^{3}-1.436^{\star} 10^{-2 *}\left(L_{d e n}-\right.
$$
$42)^{2}+0.5118^{*}\left(L_{d e n}-42\right)$

Statistical significance was set at $\alpha=0.05$. When appropriate, results are reported as an effect size with a corresponding $95 \% \mathrm{CI}$. Analyses and graphical presentation were done with SPSS v. 17 and Microsoft Excel v. 2010.

Stage 2: Estimation of disability-adjusted lifeyears (DALYs)

DALYs lost to the cases of high NA were calculated employing the methodology of WHO (1). We used the formula:

$D A L Y=$ number of people highly annoyed ${ }^{*}$ severity weight * duration of exposure

where "severity weight" corresponds to the disability weight (DW) proposed by WHO for cases with high NA, i.e., 0.02 with an uncertainty interval $0.01-0.12$, and "duration of exposure" causing high annoyance is set at 1 year.

DALYs were first computed for the total population of Plovdiv based on official road traffic noise exposure data $(55-59 \mathrm{~dB}$ to $>75 \mathrm{~dB}$ ) for 2012 , i.e., percentage and number of people exposed in each $\mathrm{L}_{\mathrm{den}} 5$ - $\mathrm{dB}$ category, extracted from the Noise Observation and Information Service for Europe (NOISE) (http://noise.eionet.europa.eu/). DALYs estimation was based on the \% $\mathrm{HA}$ at the midpoint of each $\mathrm{L}_{\text {den }}$ category. However, the \%HA in the exposure category $>75 \mathrm{~dB}$ was assumed to be the same as in the 70$75 \mathrm{~dB}$ category (1). \%HA was obtained as a function of $\mathrm{L}_{\text {den }}$ according to the two compared exposure-response curves (the linear approximation in this study and Miedema and Oudshoorn's polynomial (9)). These functions were extrapolated to the total popu- lation of Plovdiv. The main results are based on Miedema and Oudshoorn's polynomial (9) since they are considered more reliable and therefore recommended as a preliminary basis for future decision-making. As a supplementary analysis we applied Miedema and Oudshoorn's polynomial (9) to the urban population of all major Bulgarian agglomerations extracting noise exposure data $(55-59 \mathrm{~dB}$ to $>75 \mathrm{~dB})$ for 2012 from NOISE.

According to the method of Brown (19), the monetary value of DALYs lost to high NA was computed by multiplying the estimated DALYs by the Bulgarian gross domestic product per capita in Purchasing Power Standards (PPS) for 2012 (i.e., $€ 12$ 000) (20), where one PPS roughly equals one euro $(€)$. Conservative assumptions were applied to the calculations. Data were processed with Microsoft Excel v. 2010.

\section{RESULTS}

\section{Individual determinants of noise annoyance}

A total of 1906 people were approached and of those only 368 agreed to participate (response rate = 19.31\%). Main reason for non-participation was lack of time and/or fear of revealing personal information. Table 1 gives participants' characteristics. The sample is somewhat representative of the population due to higher prevalence of women and more educated people from the middle socio-economic class, which is a trend in surveys observed worldwide (16). Noteworthy, 155 (50.82\%) participants had missing data on their residential address.

NA did not differ statistically significantly across sexes $\left(\mathrm{t}_{(302)}=-0.77, \mathrm{p}=0.443\right)$, participants with a different marital status $\left(\mathrm{F}_{(3,24.12)}, \mathrm{p}=0.121\right)$ or occupation $\left(\mathrm{F}_{(3,48.46)}=2.59, \mathrm{p}=0.064\right)$. Education $\left(\mathrm{r}_{\mathrm{s}}\right.$ ${ }_{(302)}=-0.03,95 \%$ CI: $\left.-0.14,0.09\right)$ and the reported socio-economic status $\left(\mathrm{r}_{\mathrm{s}(301)}=0.02,95 \%\right.$ CI: $\left.-0.09,0.13\right)$ were not important correlates either. The linear trend with age was also negligible $\left(r_{(299)}=-0.09,95 \%\right.$ CI: $-0.20,0.03)$. However, NA had an inverse U-shaped relationship with age squared $\left(\mathrm{r}_{(299)}=-0.11,95 \% \mathrm{CI}\right.$ : $-0.22,0.001$ ) with a peak for middle-aged people. Its most influential correlate was noise sensitivity ( $\mathrm{r}$ ${ }_{(285)}=0.56,95 \%$ CI: $\left.0.48,0.64\right)$, whereas the correlation with $\mathrm{L}_{\mathrm{den}}$ modelled as an ordinal variable was only modest $\left(\mathrm{r}_{\mathrm{s}(147)}=0.24,95 \%\right.$ CI: $\left.0.09,0.39\right)$. Overall, about $16 \%$ (95\% CI: $12 \%, 21 \%)$ of the participants 
Road traffic noise and annoyance: exposure-response relationship and burden of disease calculations in Bulgaria

Table 1. Basic demographic characteristics of the participants

\begin{tabular}{lcc}
\hline Demographics & \multicolumn{2}{c}{ Valid cases } \\
Age, mean (SD) & $\begin{array}{c}39.19(14.26) ; \\
\text { range: } 18-83\end{array}$ & 302 \\
Male, n (\%) & $134(43.93)$ & 305 \\
Bulgarian, n (\%) & $260(85.25)$ & 305 \\
Education, n (\%) & & 305 \\
basic & $6(1.97)$ & \\
upper secondary & $122(40.00)$ & \\
bachelor/master & $173(56.72)$ & \\
PhD/DSc & $4(1.31)$ & \\
Marital status, n (\%) & & \\
married/spouse & $207(67.87)$ & \\
single & $74(24.26)$ & \\
widowed & $8(2.62)$ & \\
divorced & $16(5.25)$ & \\
Occupation, n (\%) & & \\
employed & $206(67.54)$ & \\
studying & $59(19.34)$ & \\
unemployed & $17(5.57)$ & \\
retired & $22(7.21)$ & \\
Socio-economic status, n $\%)$ & \\
lower & & \\
middle & & \\
upper & & \\
\hline \hline
\end{tabular}

(including those with missing data) were highly annoyed by road traffic noise. Table 2 displays the \%HA across different noise bands. As can be seen, higher noise levels generally resulted in higher \%HA. Based on their mean NA scores $(M=5.33, S D=1.94$ vs. $M$ $\left.=5.54, \mathrm{SD}=2.30 ; \mathrm{t}_{(289.57)}=-0.85, \mathrm{p}=0.396\right)$ participants with missing data on their residential address $\left(\mathrm{L}_{\text {den }}\right.$ respectively) did not differ from the rest.

\section{Exposure-response relationship}

A bivariate logistic regression analysis was conducted to establish the exposure-response relationship between NA and $\mathrm{L}_{\text {den }}$. The model contained $\mathrm{L}_{\text {den }}$ as a continuous predictor and the \%HA as a dichotomized outcome. The Hosmer-Lemeshow test suggested reasonable fit of the model to the data $\left(\chi_{(2)}^{2}\right.$ $=4.29, \mathrm{p}=0.117)$ but the predictive power was low (Nagelkerke's $\left.\mathrm{R}^{2}=0.03\right)$. The estimated intercept
Table 2. Distribution of highly annoyed people across different noise bands in the sample

\begin{tabular}{|l|c|c|c|c|}
\hline $\mathrm{L}_{\mathrm{den}}[\mathrm{dB}(\mathrm{A})]$ & \multicolumn{2}{|c|}{$\begin{array}{c}\text { People highly } \\
\text { annoyed }\end{array}$} & \multicolumn{2}{c|}{$\begin{array}{c}\text { People in the } \\
\text { category }\end{array}$} \\
\hline & $\mathbf{n}$ & $\%$ & $\mathbf{n}$ & $\%$ \\
\hline $50-55$ & 0 & 0.00 & 3 & 2.01 \\
$55-60$ & 1 & 6.66 & 15 & 10.07 \\
$60-65$ & 7 & 24.13 & 29 & 19.46 \\
$65-70$ & 7 & 12.96 & 54 & 36.24 \\
$70-75$ & 13 & 29.55 & 44 & 29.53 \\
$75-80$ & 1 & 25.00 & 4 & 2.68 \\
Total & 29 & 19.46 & 149 & 100.00 \\
\hline \hline
\end{tabular}

Note. Figures are based on cases without missing data.

and the slope were $-6.329(\mathrm{SE}=2.883, \mathrm{p}=0.028)$ and 0.073 (SE $=0.042, \mathrm{p}=0.085$ ), respectively. Sensitivity analysis was conducted to address the smallish sample size and low number of cases in the lowest and highest exposure categories. The results of the regression model did not change noticeably after imputing 50 datasets and re-running the regression. Figure 1 depicts the logistic prediction function along with its linear approximation based on the survey data points in the range $50-80 \mathrm{~dB}$ :

$\% H A=-51.328+1.0417^{*} L_{d e n}$

A comparison between the estimated prediction curve derived from the logistic regression model and Miedema and Oudshoorn's polynomial (9) for road traffic noise is given in Figure 2. Miedema and Oudshoorn's curve (9) fell within the 95\% CI of our logistic prediction curve. However, both the 95\% CI and $95 \%$ PI of the latter were wide probably due to the few observations from which the function was derived. Both functions were extrapolated to the range $40-90 \mathrm{~dB}$ in the graph, but the logistic curve can only be trusted in the $55-75 \mathrm{~dB}$ range. The linear approximation was used in DALYs calculation since approximations are easier to use than the model itself (9).

\section{DALYs lost to high noise annoyance}

Table 3 presents the results of DALYs computation for the city of Plovdiv. As evident, the two exposure-response functions generated very similar estimates. DALYs are given along with their cred- 
Angel Dzhambov, Donka Dimitrova

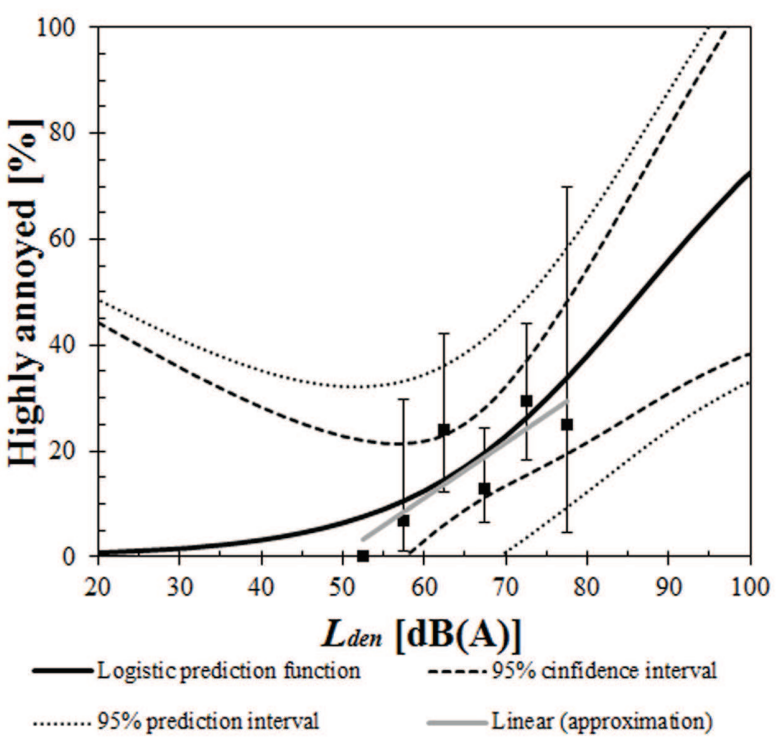

Fig. 1. Logistic prediction function and its linear approximation for the percentage highly annoyed people

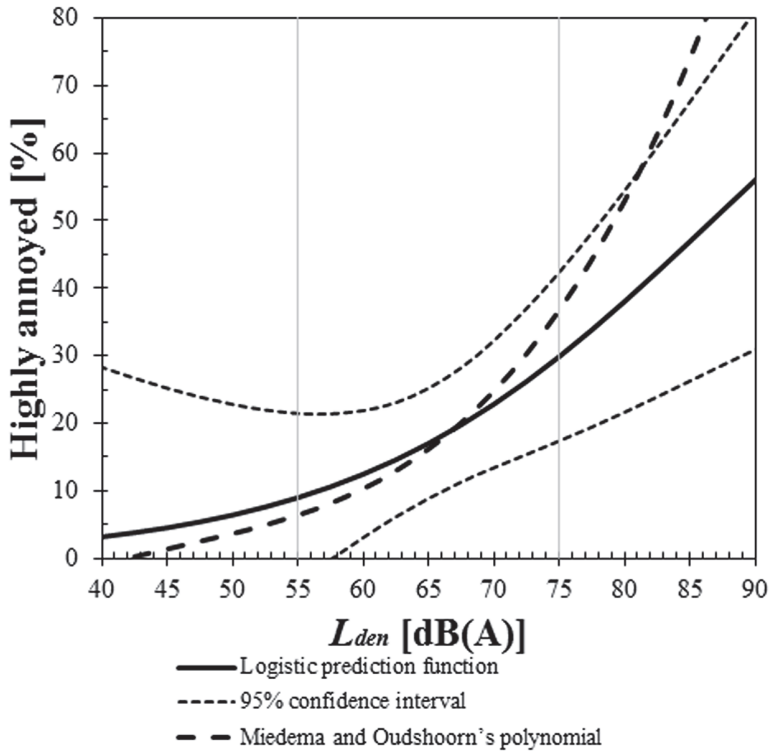

Fig. 2. Comparison of exposure-response relationships between road traffic noise and the percentage highly annoyed people

ible range according to the assumption that DW = 0.02 and taking its extremes: $0.01-0.12$. The linear approximation yielded an annual loss of 1119 DALYs which translated into about $€ 13.42$ million. This was similar to the estimated with Miedema and Oudshoorn's polynomial (9) (DALYs = 1188.17 and $€ 14.26$ million). As a secondary analysis, Miedema and Oudshoorn's polynomial (9) was applied to the urban population of all major Bulgarian agglomera- tions (approximately 2568250 people according to NOISE) yielding an estimated loss of 6399.60 DALYs (3 199.80 - 38 397.60) and $€ 76795207.93$ (38 397 603.96 - 460771247.60$)$, respectively.

\section{DISCUSSION}

\section{Key findings}

This study stems from our endevour to quantify the socio-economic burden of road traffic noise in Bulgaria. While our field survey, which supplied data for the construction of an exposure-response function, had some prominent limitations, the linear approximation of the latter yielded very close estimates to Miedema and Oudshoorn's polynomial (9) in the range $55-75 \mathrm{~dB}$. This gives us reason to recommend Miedema and Oudshoorn's curve (9) for further quantification of the impact of road traffic noise in Bulgaria. Burden of disease analyses showed that severe NA in Plovdiv was associated with an annual loss of 1 188.17 DALYs and about $€ 14.26$ million. On a national scale these figures are even more alarming.

As far as we are aware, this study adds to the state of current knowledge about the socio-economic burden of road traffic noise in Bulgaria. Recent research has found a suspected $€ 11.6$ million annual loss to road traffic noise-attributed myocardial infarction in the country (6). High NA had a much larger impact in just one major agglomeration. This research is also in line with the current academic development in this field of noise hygiene and epidemiology in South-East Europe $(21,22)$.

\section{Limitations}

The study has two sets of limitations. The first stems from the filed survey we carried out, namely, the low response rate and partial representativeness of the sample. These issues are not uncommon in epidemiological research especially given the poor survey culture and sense of personal salience of environmental issues in Bulgaria. Also, people living under severe noise stress might be blaming the latter for their psychological and somatic health problems and thus be more likely to participate in environmental surveys as demonstrated by the skew of the sample towards highly exposed people in comparison to the estimated proportions across different noise bands in the agglomeration (Compare Table 2 and Table 3).

The sample size (after excluding cases with missing data) resulted in wide confidence intervals 
Road traffic noise and annoyance: exposure-response relationship and burden of disease calculations in Bulgaria

Table 3. Highly annoyed people across different noise bands in Plovdiv and corresponding disability-adjusted life years (comparison of exposure-response functions)

\begin{tabular}{|c|c|c|c|c|c|c|c|}
\hline \multirow{2}{*}{$\begin{array}{l}\text { Exposure } \\
\text { category } \\
\mathrm{L}_{\mathrm{den}} \\
{[\mathrm{dB}(\mathrm{A})]}\end{array}$} & \multicolumn{2}{|c|}{$\begin{array}{l}\text { Number of peo- } \\
\text { ple exposed in the } \\
\text { population }\end{array}$} & \multicolumn{2}{|c|}{$\begin{array}{l}\text { Number of people } \\
\text { highly annoyed in } \\
\text { the category }\end{array}$} & \multicolumn{3}{|c|}{ DALYs in the population } \\
\hline & $\mathrm{n}$ & $\%^{a}$ & $\mathrm{n}$ & $\%$ & $\mathrm{DW}=0.01$ & $\mathrm{DW}=0.02$ & $\mathrm{DW}=0.12$ \\
\hline \multicolumn{8}{|c|}{ Linear approximation in this study } \\
\hline $55-60$ & 67800 & 19.83 & 5810 & 8.57 & 58.10 & 116.21 & 697.23 \\
\hline $60-65$ & 105500 & 30.86 & 14536 & 13.78 & 145.36 & 290.72 & 1744.33 \\
\hline $65-70$ & 117100 & 34.25 & 22233 & 18.99 & 222.33 & 444.67 & 2668.02 \\
\hline $70-75$ & 54600 & 15.97 & 13211 & 24.20 & 132.11 & 264.21 & 1585.27 \\
\hline$>75$ & 600 & 0.18 & 145 & $24.20^{\mathrm{b}}$ & 1.45 & 2.90 & 17.42 \\
\hline Total & \multicolumn{2}{|l|}{345600} & \multicolumn{2}{|c|}{55936} & 559.36 & 1119.00 & 6712.27 \\
\hline \multicolumn{5}{|c|}{$\begin{array}{l}\text { Monetary valuation of DALYs lost } \\
\text { (in Purchasing Power Standards euro) }\end{array}$} & 6712272.78 & 13424545.56 & 80547273.36 \\
\hline \multicolumn{8}{|c|}{ Miedema and Oudshoorn's polynomial ${ }^{[9]}$} \\
\hline $55-60$ & 67800 & 19.83 & 5531 & 8.16 & 55.31 & 110.62 & 663.70 \\
\hline $60-65$ & 105500 & 30.86 & 13671 & 12.96 & 136.71 & 273.42 & 1640.55 \\
\hline $65-70$ & 117100 & 34.25 & 23509 & 20.08 & 235.09 & 470.18 & 2821.05 \\
\hline $70-75$ & 54600 & 15.97 & 16516 & 30.25 & 165.16 & 330.33 & 1981.95 \\
\hline$>75$ & 600 & 0.18 & 181 & $30.25^{\mathrm{b}}$ & 1.81 & 3.63 & 21.78 \\
\hline Total & \multicolumn{2}{|l|}{345600} & \multicolumn{2}{|c|}{59409} & 594.09 & 1188.17 & 6129.04 \\
\hline \multicolumn{5}{|c|}{$\begin{array}{l}\text { Monetary valuation of DALYs lost } \\
\text { (in Purchasing Power Standards euro) }\end{array}$} & 7129040.34 & 14258080.67 & 85548484.03 \\
\hline
\end{tabular}

Note. Data are extracted from Noise Observation and Information System for Europe and refer to 2012. Exposure data for $<55 \mathrm{~dB}$ is not available. Monetary value of one DALY is $€ 12$ 000. Duration of exposure is assumed to be 1 year. The figures are rounded. ${ }^{a}$ Extracted percentages do not add-up exactly to $100 \%$ probably due to rounding error. ${ }^{b}$ The percentage of people highly annoyed in the exposure category $>75 \mathrm{~dB}$ is assumed to be the same as in the 70-75 $d B$ category [1].

$D W$ - disability weight, DALYs - disability-adjusted life years, $L_{\text {den }}$-day-evening-night equivalent sound level

for the prediction function. On the other hand, imputation of these missing values did not change it considerably. The fact that we did not have a truly continuous noise exposure variable resulted in a small number of data points. The low \%HA people in the $>75 \mathrm{~dB}$ category could be explained by the selfselection of people of low noise sensitivity (1). In the logistic regression, however, the problems with the low number of cases in the lowest and highest categories were attenuated to some extent by treating $\mathrm{L}_{\text {den }}$ as a continuous variable.

One should also bear in mind that the NA question in the survey asked about general NA at home and not specifically road traffic NA. Nevertheless, the close fit of the exposure-response function to $\mathrm{Mi}$ edema and Oudshoorn's polynomial suggests that road traffic is probably the main cause for NA in the city; furthermore, our logistic model coefficients are remarkably similar to those of a Hong Kong study (23). In that study $L_{\text {den }}$ also explained a small proportion of the variance in NA. Given its large dataset and the comparable noise exposure and NA assessment methodology we believe this implies at least some degree of external validity of our findings.

Despite these limitations we believe that their impact is not crucial since the field survey results only reinforce the main results based on official noise exposure data and Miedema and Oudshoorn's 
Angel Dzhambov, Donka Dimitrova

polynomial (9). That is, quantification of the burden of disease is a stand-alone research which does not necessarily call for a prior validation of the exposureresponse relationship $(1,6)$.

The WHO methodology has its inherent limitations as well: it is associated with various uncertainties regarding the validity and generalizability of the exposure-response function, disability weights, quality and accuracy of noise mapping, criticism of DALYs, etc. $(1,6)$. Those are, however, justified by WHO experts (1) and outweighed by the critical need for a quantitative burden of disease assessment in order to guide future noise legislation. One need not have $100 \%$ evidence of everything in order to act on the available evidence (24). Thus, the reported results are tentative but highly warranted and give a feel of the extent to which road traffic noise affects our society and healthcare system.

\section{Future research}

Future research should extend the WHO methodology to the whole urban population in Bulgaria and focus on other health endpoints such as tinnitus, sleep disturbance, cognitive impairment, etc. A multi-centre population-based survey overcoming the limitations of our filed study is mandatory in order to derive precise exposure-response curves not only for NA, but for sleep disturbance and cardiovascular endpoints. In regards to NA one might consider alternative approaches for quantification of its socio-economic impact (25).

Environmental health policy should focus on all dimensions of noise pollution while local authorities continue their active involvement in road traffic noise control. Finally, adequate training in environmental health of primary public health professionals (26) is needed to facilitate dissemination of information for person-level interventions (e.g., window closing habits, sound insulation, hearing protection during night, orientation of rooms in the dwelling, stress management, etc.) among vulnerable groups and individuals.

\section{CONCLUSIONS}

The exposure-response relationship between road traffic noise and annoyance in this study closely resembled Miedema and Oudshoorn's polynomial in the range $55-75 \mathrm{~dB}$. Based on the latter we estimated 1 188.17 DALYs and $€ 14.26$ million lost annually in Plovdiv and about 6399.60 DALYs (€ 76.80 million) in Bulgaria. Road traffic NA appears to have a considerable socio-economic impact in the country which calls for decisive actions by involved authorities. Future use of Miedema and Oudshoorn's polynomial is substantiated.

\section{REFERENCES}

1. World Health Organization (WHO). Burden of Disease from Environmental Noise, Regional Office for Europe; 2011.

2. Basner M, Babisch W, Davis A, et al. Auditory and non-auditory effects of noise on health. Lancet. 2014;383(9925):1325-32.

3. Heinonen-Guzejev M. Noise sensitivity - medical, psychological and genetic aspects [dissertation]. University of Helsinki: Helsinki University Printing House Helsinki; 2008.

4. Guski R. Personal and social variables as co-determinants of noise annoyance. Noise Health. 1999;1:45-56.

5. Ndrepepa A, Twardella D. Relationship between noise annoyance from road traffic noise and cardiovascular diseases: a meta-analysis. Noise Health. 2011;13:251-259.

6. Dzhambov AM, Dimitrova DD. Evaluation of the social and economic burden of road traffic noise-attributed myocardial infarction in Bulgarian urban population. Arh Hig Rada Toksikol. 2015;66(1):15-21.

7. Monitor. "Noise terrorizes two million Bulgarians". [Newspaper article from September 17, 2014]. Available at: http://www.webcitation. org/6WMOVXGnk. Accessed: February 12, 2015. Bulgarian.

8. Laszlo HE, McRobie ES, Stansfeld SA, et al. Annoyance and other reaction measures to changes in noise exposure - a review. Sci Total Environ. 2012;435-436: 551-62.

9. Miedema HM, Oudshoorn CG. Annoyance from transportation noise: relationships with exposure metrics DNL and DENL and their con- 
Road traffic noise and annoyance: exposure-response relationship and burden of disease calculations in Bulgaria

fidence intervals. Environ Health Perspect. 2001;109(4):409-16.

10. Miedema HM, Vos H. Noise annoyance from stationary sources: relationships with exposure metric day-evening-night level (DENL) and their confidence intervals. J Acoust Soc Am. 2004;116:334-43

11. Takuchev N, Vasileva I, Petrova S. Dispersion Modeling of the Air Pollution, Emitted by the Traffic in the Transport Tunnel under the Old Town of Plovdiv, Bulgaria. Ecol Balk. 2014;6:73-86.

12. Dzhambov AM, Dimitrova DD. Validating a short Bulgarian version of a psychometric instrument for multidimensional noise sensitivity assessment. Folia Med (Plovdiv). 2014;56(2):116-25.

13. Pedersen TH. The "Genlyd" Noise Annoyance Model. Dose-Response Relationships Modelled by Logistic Functions, AV 1102/07. DELTA, 2007. Available at: http://share.madebydelta.com/wpcontent/publications/akustik/paper_og_rapport/ The_Genlyd_Noise_Annoyance_Model.pdf. Accessed: February 18, 2015.

14. Agay-Shay K, Peled A, Crespo AV, Pet al. Green spaces and adverse pregnancy outcomes. Occup Environ Med. 2014;71:562-9.

15. Directive 2002/49/EC of the European Parliament and of the Council of 25 June 2002 relating to the assessment and management of environmental noise. Off J Eur Commun. 2002;L189:12-25.

16. Galea S, Tracy M. Participation rates in epidemiologic studies. Ann Epidemiol. 2007;17:643-53.

17. Altman DG, Gardner MJ. Regression and Correlation. In: Altman DG, Machin D, Bryant TN, Gardner MJ. (Eds.). Statistics with Confidence (2nd Ed.). BMJ Books; 2000.

18. Newcombe, Robert G. Two-Sided Confidence Intervals for the Single Proportion: Comparison of Seven Methods. Stat Med. 1998;17: 857-72.

19. Brown DW. Economic value of disability-adjusted life years lost to violence: estimates for WHO Member States. Rev Panam Salud Publica. 2008;24(3):203-9.

20. European Commission, EUROSTAT. GDP and beyond, Measuring quality of life in the EU; 2014. Available at http://epp.eurostat.ec.europa.eu/cache/ ITY_PUBLIC/3-19032014-CP/EN/3-19032014-CPEN.PDF. Accessed: July 22, 2014.
21. Paunovic K, Belojević G. Burden of myocardial infarction attributable to road-traffic noise: A pilot study in Belgrade. Noise Health. 2014;16:374-9.

22. Ristovska G, Gjorgjev D, Polozhani A, et al. Environmental noise and annoyance in adult population of Skopje: a cross-sectional study. Arh Hig Rada Toksikol. 2009;60(3):349-55.

23. Environmental Protection Department, Hksar Government. Final report for the provision of service for the study of health effects of transportation noise in Hong Kong; 2012.

24. World Health Organization (WHO). Evaluation and Use of Epidemiological Evidence for Environmental Health Risk Assessment. Guideline document. Copenhagen: WHO Regional Office for $\mathrm{Eu}-$ rope; 2000.

25. Gjestland T. The socio-economic impact of noise: a method for assessing noise annoyance. Noise Health. 2007;9(35):42-4.

26. Training of Professionals in Environment and Health. Public Health Services Gelderland Midden. Published by European Commission Directorate General for Health and Consumers, Luxembourg; 2011. 